\title{
Dynamic testing of asphalt mixes
}

\author{
Jean-Claude Carret $^{1, *}$, Hervé Di Benedetto ${ }^{1}$, and Cédric Sauzéat ${ }^{1}$ \\ ${ }^{1}$ University of Lyon / ENTPE, LTDS (UMR CNRS 5513), Rue M. Audin, 69518, Vaulx en Velin, France
}

\begin{abstract}
In the presented research, conventional cyclic tension-compression complex modulus tests and dynamic tests were performed on an asphalt mix (AM) specimen. For the tension-compression tests, the complex modulus was calculated from the measurements of the axial strain and axial stress. For the dynamic tests, an automated impact hammer equipped with a load cell and an accelerometer were used to determine the frequency response functions (FRFs) at five different temperatures. A back-analysis using finite element method (FEM) calculations and a very simple modelling of the material behaviour was proposed to determine the complex modulus of the specimen at each tested temperature. Complex modulus results from dynamic and cyclic tests were compared and are in good agreement. The norm of the complex modulus obtained from dynamic test is slightly higher and no significant difference is seen for the phase angle. Part of the differences observed may be explained by the nonlinearity of AM (strain amplitude is about 500 times smaller for dynamic tests).
\end{abstract}

\section{Introduction}

Asphalt mixes (AM) have a linear viscoelastic (LVE) behaviour in the small strain domain [1]. Their properties are strongly dependent on frequency and temperature and the maximum ratio of modulus values can be up to one thousand. Therefore, traditional elastic analyses are not pertinent with AM. Cyclic tension-compression complex modulus tests are traditionally used to determine the LVE properties of AM but these tests require expensive experimental devices such as hydraulic presses and are not applicable in situ. Non-destructive dynamic tests are an economic alternative. They are simple to perform and possibly adaptable for measurements on pavement structures. Impulse techniques using impact loadings [2,3] are known to provide accurate characterization of material properties in the case of elastic materials $[4,5]$. In the case of LVE materials, dynamic tests could be a great alternative to conventional cyclic tension-compression tests. Recently, measurement of frequency response functions (FRFs) have been performed on AM [6-11]. Gudmarsson et al. [7, 8] and Carret et al. [9, 10] showed that using FRFs measurements to obtain the LVE properties of AM is a very promising approach. In this paper, the possibility of using a simple approach using finite element calculations to obtain the complex modulus of the material at each temperature from FRFs measurements is studied. The accuracy of the proposed approach is evaluated by comparing the results obtained from dynamic tests with results obtained from more conventional cyclic tension-compression tests. First, the material tested in this study is presented. Then, the LVE behaviour characterization with cyclic tensioncompression tests is introduced. Next, dynamic tests are introduced and the proposed approach to back-analyse the dynamic measurements is explained. Finally, complex modulus data from tension-compression tests are compared with complex modulus results obtained with the proposed back-analysis of the dynamic tests.

\section{Materials}

The material tested in this study is a laboratory designed mix that was used in the IMPROVMURE project [12] from the French national research agency. It was fabricated using bitumen foam and it has a reclaimed asphalt pavement (RAP) content of $70 \%$. More details about the specimen considered in this study are listed in Table 1.

Table 1. Details of the material used in this study.

\begin{tabular}{|c|c|c|c|c|}
\hline $\begin{array}{c}\text { Mass } \\
(\mathbf{g})\end{array}$ & $\begin{array}{c}\text { Height } \\
\mathbf{( m m})\end{array}$ & $\begin{array}{c}\text { Diameter } \\
\mathbf{( m m )}\end{array}$ & $\begin{array}{c}\text { Density } \\
\mathbf{( k g / \mathbf { m } ^ { 3 }}\end{array}$ & $\begin{array}{c}\text { Void } \\
\text { Ratio } \\
\mathbf{( \% )}\end{array}$ \\
\hline 1330 & 123 & 75 & 2449 & 3.8 \\
\hline
\end{tabular}

\section{Linear viscoelastic (LVE) behaviour characterization: cyclic tension- compression tests}

The linear viscoelastic (LVE) behaviour is commonly characterized using cyclic tension-compression complex modulus tests. They consist in applying cyclic sinusoidal axial loadings at various temperatures (from $-25^{\circ} \mathrm{C}$ to $55^{\circ} \mathrm{C}$ ) and frequencies (from $0.003 \mathrm{~Hz}$ to $10 \mathrm{~Hz}$ ) using a hydraulic press in strain-controlled mode. The applied strain amplitude is around $50 \mu \mathrm{m} / \mathrm{m}$ and the axial strain $\varepsilon_{z}$

* Corresponding author: jean-claude.carret@entpe.fr 
is measured with three extensometers placed at $120^{\circ}$ from each other. The resulting axial stress $\sigma_{z}$ is measured with a load cell. The radial strain $\varepsilon_{\mathrm{r}}$ can be derived from the measurements of two non-contact sensors but it was not considered in this study. The procedure developed at ENTPE/University of Lyon laboratory is detailed in other publications [13-16] and the test set-up is illustrated in Fig. 1.

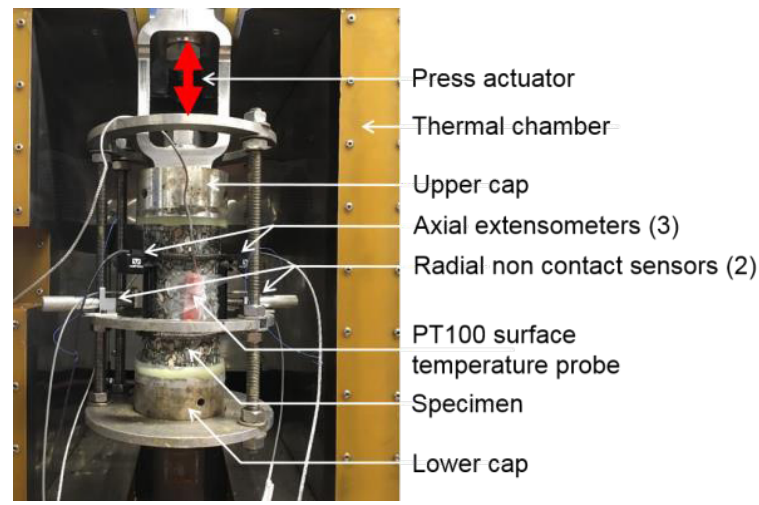

Fig. 1. Tension-compression test set-up (ENTPE laboratory, University of Lyon).

The complex notation of the axial stress and the axial strain are given in equation (1):

$$
\begin{aligned}
& \sigma_{z}^{*}=\sigma_{0 z} \cdot e^{j \omega t} \\
& \varepsilon_{z}^{*}=\varepsilon_{0 z} \cdot e^{j\left(\omega t+\varphi_{\varepsilon z}\right)}
\end{aligned}
$$

where $\omega$ is the pulsation $(\omega=2 \pi f$, where $f$ is the frequency), $\sigma_{0 z}$ is the norm of the complex axial stress and $\varepsilon_{0 z}$ and $\varphi_{\varepsilon z}$ are the norm and phase angle of the complex axial strain. An example of the experimental data of the axial strain and axial stress is given in Fig. 2 for two loading cycles at $15^{\circ} \mathrm{C}$ and $1 \mathrm{~Hz}$.

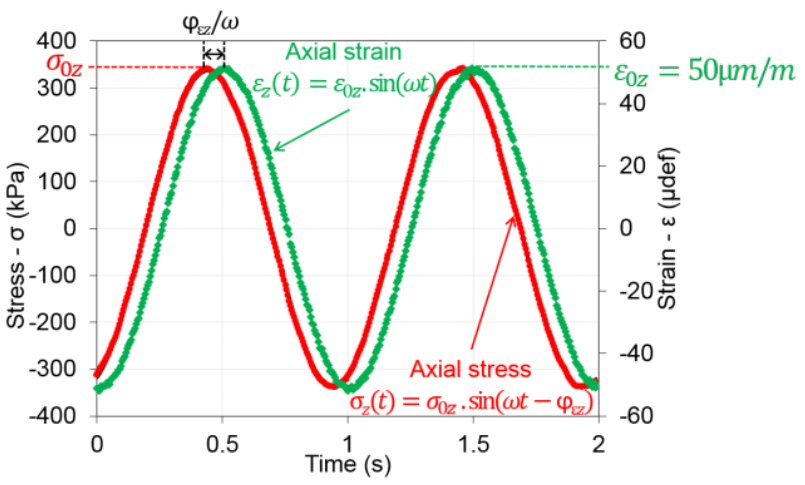

Fig. 2. Tension-compression experimental data (axial strain, axial stress and radial strain) for two loading cycles at $15^{\circ} \mathrm{C}$ and $1 \mathrm{~Hz}$.

The complex modulus is defined as the ratio between the axial stress and the axial strain (in complex notation) and it is calculated at each frequency and temperature according to equation (2):

$$
E^{*}=\frac{\sigma_{z}^{*}}{\varepsilon_{z}^{*}}=\frac{\sigma_{0 z}}{\varepsilon_{0 z}} \cdot e^{-j \varphi_{\varepsilon z}}=\left|E^{*}\right| \cdot e^{j \varphi_{E}}
$$

where $\mathrm{E}^{*}$ is the norm of the complex modulus and $\varphi_{\mathrm{E}}$ is the phase angle of the complex modulus.

For most of the bituminous materials, the time temperature superposition principle (TTSP) is verified in the linear and nonlinear domains [17-19]. Therefore, it is possible to generate master curves at one reference temperature $\left(\mathrm{T}_{\mathrm{ref}}\right)$ by shifting experimental data on the frequency axis. The shifted frequencies are called reduced frequencies $\left(f_{\text {red }}\right)$, which are the frequencies of the other temperatures $\left(\mathrm{f}_{\mathrm{T}}\right)$ multiplied by shift factors $\left(\mathrm{a}_{\mathrm{T}}\right)$ that depend on the temperature $\mathrm{T}$ :

$$
f_{\text {red }}=a_{T}(T) \cdot f_{T}
$$

The variation of the shift factors with temperature can be described with the Williams-Landel-Ferry (WLF) equation [20]:

$$
\log \left(a_{T}\right)=-\frac{C_{1}\left(T-T_{r e f}\right)}{C_{2}+T-T_{r e f}}
$$

where $\mathrm{C}_{1}$ and $\mathrm{C}_{2}$ are the two constants of the WLF equation and $\mathrm{T}_{\mathrm{ref}}$ is the reference temperature.

\section{Dynamic tests}

Dynamic tests are economic tests and simple to perform in comparison with cyclic tension-compression tests. In this section the experimental set-up of the dynamic tests is first introduced. Then, the methodology to obtain the LVE properties from experimental measurements is explained.

\subsection{Experimental measurement of the Frequency Response Functions (FRFs)}

First, the upper and lower metallic caps glued for the tension-compression tests were sawed before performing the dynamic measurements on the same specimen. An impact hammer equipped with a load cell (PCB model 086E80) was used to hit the cylindrical specimen in the middle of one short side (see Fig. 3). The impact hammer was automated to improve the repeatability of the test and to allow measurements directly inside the thermal chamber. The order of magnitude of the maximum strain induced in the specimen by the impact is about $0.1 \mu \mathrm{m} / \mathrm{m}$ [8-10]. The response of the material was recorded with an accelerometer (PCB model 353B15) glued in the middle of the opposite short side of the specimen (see Fig. 3). To achieve free boundary conditions, soft foam was placed under the specimen during the test. The test set-up is illustrated in Fig. 3. 


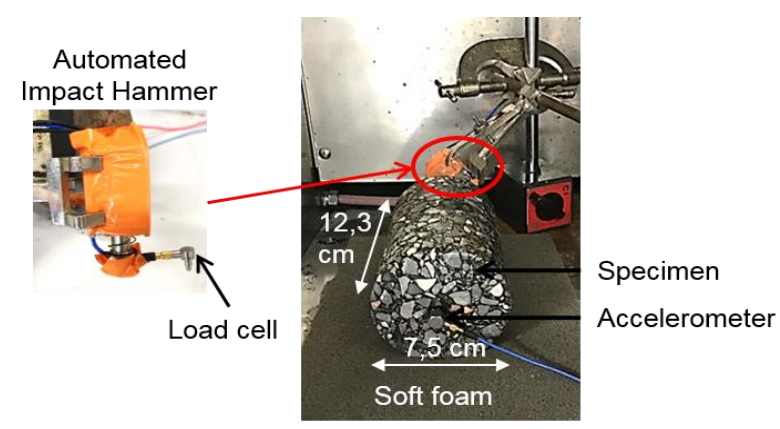

Fig. 3. Test set up for the dynamic impact tests (ENTPE laboratory, University of Lyon).

The impact hammer and the accelerometer were connected to a signal conditioner (PCB model 482C15) itself connected to a data acquisition device (NI USB6356) connected to a computer. The applied force and the acceleration were recorded with a sampling frequency of $1 \mathrm{MHz}$ by using a MATLAB application which was specifically developed for this test. The test was repeated at five temperatures $\left(-20,0,15,35\right.$ and $\left.50{ }^{\circ} \mathrm{C}\right)$ and five impacts were applied at each temperature. Fig. 4 shows an example of the time domain signals for the five hits at $15^{\circ} \mathrm{C}$.
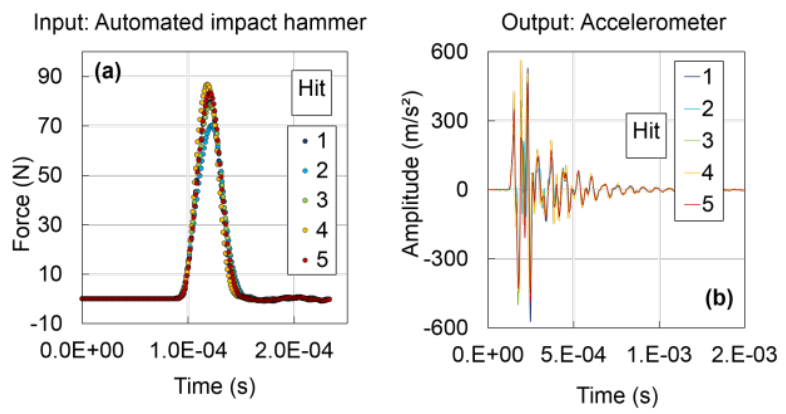

Fig. 4. Experimental data for the dynamic testing (5 hits). (a) Force in time domain; (b) Acceleration in time domain.

The experimental data in time domain were then converted in frequency domain with a $1 \mathrm{~Hz}$ resolution using the Fast Fourier Transform (FFT). The FRFs were calculated from the frequency domain signals as follow:

$$
H(f)=\left(\overline{Y(f) \cdot X^{*}(f)}\right) /\left(\overline{X(f) \cdot X^{*}(f)}\right)
$$

where $\mathrm{H}$ is the FRF, $\mathrm{Y}$ is the FFT of the measured acceleration, $\mathrm{X}$ is the FFT of the applied force, $\mathrm{X}^{*}$ is the complex conjugate of the FFT of the applied force and the bar above corresponds to the arithmetic average from the five impacts. The amplitude of the FRFs calculated at the five tested temperatures according to equation (5) are plotted in Fig. 5. It is observed in Fig. 5 that the resonance frequency and the amplitude at the resonance decrease when temperature increases, which indicate changes of material properties because of the viscoelastic behaviour of the specimen.

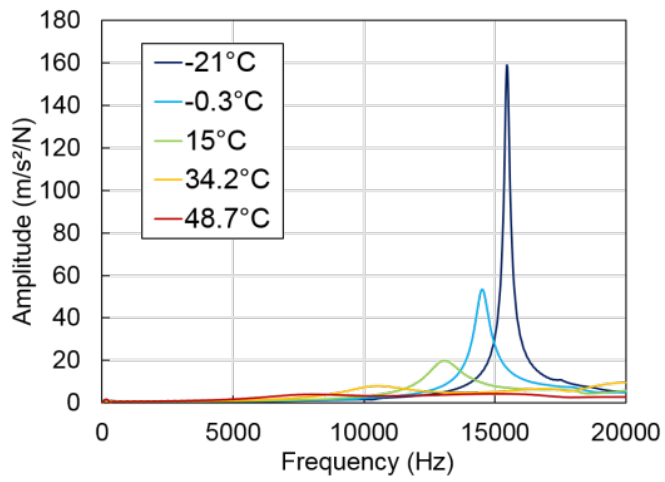

Fig.5. FRFs at the five temperatures of measurement (average of the five hits).

\subsection{FRFs from the finite element method (FEM)}

Numerical FRFs were calculated with the finite element method (FEM) considering the dynamic test boundary conditions (free boundary conditions everywhere except at the position of the impact). Fig. 6 shows the FEM mesh used for the FEM calculation of the FRFS.

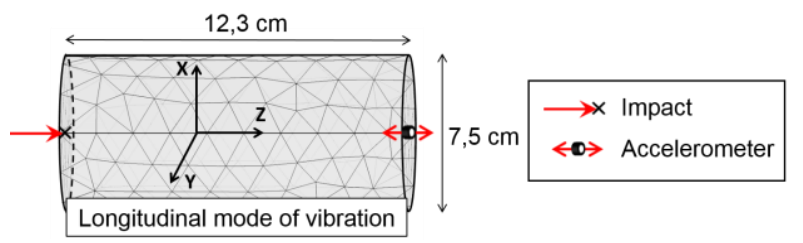

Fig. 6. Finite element model mesh. The positions of the impact and of the accelerometer correspond to the positions of the impact and of the accelerometer in the experimental tests.

The LVE behaviour of the material was simply modelled with a constant complex modulus independent of the frequency and temperature and a Poisson's ratio fixed at 0.3:

$$
\begin{aligned}
& E^{*}=E . e^{j \varphi} \\
& v=0.3
\end{aligned}
$$

where $\mathrm{E}$ and $\varphi$ are the norm and phase angle of the complex modulus. Finally, numerical FRFs were calculated at the desired frequencies by resolving the following three-dimensional equation of motion in frequency domain:

$$
-\rho \omega^{2} \mathbf{u}-\nabla \cdot \sigma=0
$$

where $\rho$ is the bulk density of the material, $\omega$ is the angular frequency, $\mathbf{u}$ is the displacement vector, $\nabla$ is the gradient tensor operator and $\sigma$ is the Cauchy stress tensor. Note that in the numerical simulations, the force applied in experimental tests at the position of the impact is replaced by a unity load. Consequently, the calculated FRFs directly correspond to the calculated accelerations in direction $\mathrm{Z}$ (direction of vibration of the accelerometer in physical tests). 


\subsection{Complex modulus from the first resonance peak only}

Carret et al. [10] showed that the value of the Poisson's ratio has a very small influence on the FRFs calculation and that the norm and phase angle of the complex modulus can be determined from FRFs measurement at each temperature with a simple process described in Fig. 7. First, the measured fundamental resonance frequency $\left(f_{1}\right)$ is used to back-calculate by dichotomy the norm of the complex modulus so that the fundamental resonance frequency of the calculated FRFs matches $f_{1}$. Then, the phase angle of the complex modulus is determined similarly, so that the measured amplitude at the fundamental resonance frequency $\left(\mathrm{A}_{1}\right)$ and the calculated one matches. With this approach, one value of the complex modulus is back-calculated from dynamic tests at the first resonance frequency for each tested temperature.

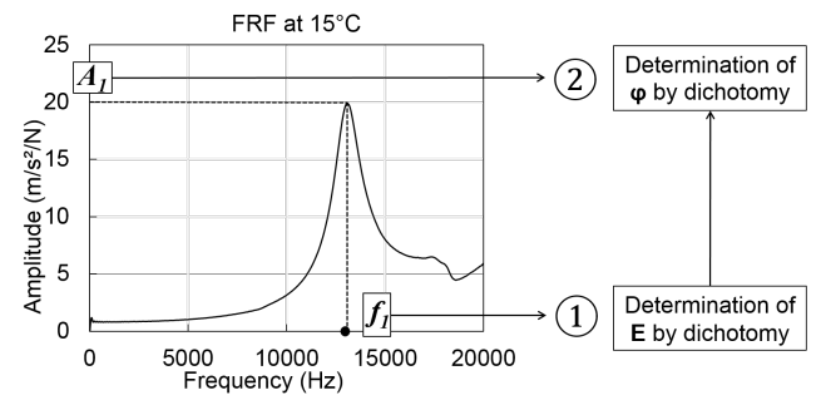

Fig. 7. Principle of the determination of the LVE properties $\mathrm{E}$ and $\varphi$ at each temperature (example at $15^{\circ} \mathrm{C}$ ).

\section{Results and analysis}

The values of the norm and phase angle of the complex modulus determined from dynamic tests at the five temperatures of measurement are listed in Table 2. The norm (resp. the phase angle) of the complex modulus decreases (resp. increases) as temperature increases. This is an expected result due to the viscoelastic behaviour of the material. When temperature increases, the damping in the specimen increases and the viscous component of the behaviour is more important. As a result, the phase angle of the complex modulus increases and the norm of the complex modulus decreases.

Table 2. LVE properties $\mathrm{E}$ and $\varphi$ obtained from the dynamic tests at the five tested temperatures.

\begin{tabular}{|c|c|c|c|c|c|}
\hline $\begin{array}{c}\text { Temperature } \\
\left({ }^{\circ} \mathbf{C}\right)\end{array}$ & -21 & -0.3 & 15 & 34.2 & 48.7 \\
\hline $\mathbf{E}(\mathbf{M P a})$ & 37217 & 32793 & 26567 & 17153 & 9498 \\
\hline $\boldsymbol{\varphi}\left({ }^{\circ}\right)$ & 0.61 & 1.82 & 4.79 & 11.82 & 22.50 \\
\hline
\end{tabular}

The results obtained from dynamic tests were compared with the results of the cyclic tensioncompression tests. The master curves at $15^{\circ} \mathrm{C}$ of the norm and phase angle of the complex modulus determined from the two tests are plotted in Fig. 8. The constants of the WLF equation determined from the tension-compression tests $\left(\mathrm{C}_{1}=24.9\right.$ and $\mathrm{C}_{2}=166.6$ at $\left.15^{\circ} \mathrm{C}\right)$ were also used to shift the data from dynamic tests. A good proximity between the results from dynamic and cyclic tests is seen in Fig. 8, both for the norm and phase angle of the complex modulus. The norm of the complex modulus determined from dynamic tests is slightly higher and the difference tends to increase with temperature (or when reduced frequencies decrease). However, the difference remains less than about $15 \%$ on the whole considered reduced frequency range. For the phase angle, the results from both tests are almost overlaid and no significant difference can be seen.

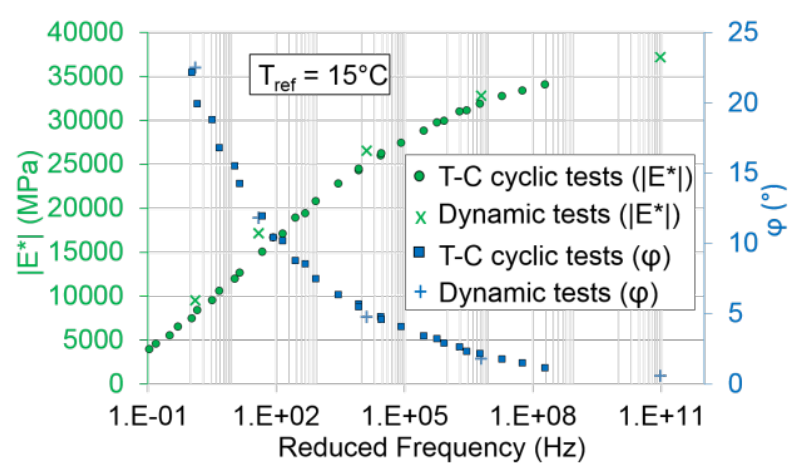

Fig.8. Master curves of the norm and phase of the complex modulus for the tension-compression and the dynamic tests.

As a reminder, the applied strain amplitude is approximately 500 times lower in dynamic tests than in tension-compression tests. Due to the nonlinear behaviour of AM with the strain amplitude [21-23], the norm of the complex modulus obtained from dynamic tests is expected to be higher than the norm of the complex modulus obtained from tension-compression tests. Therefore, nonlinearities may explain at least part of the differences observed between dynamic and cyclic tests results. The previous observations demonstrate that dynamic tests are a promising approach to accurately characterize the LVE behaviour of AM over a wide reduced frequency range.

\section{Conclusion}

In this paper, conventional cyclic tension-compression tests and dynamic tests were performed to characterize the LVE behaviour of an AM specimen. The experimental measurements from dynamic tests were back-analysed with a simple procedure to determine one value of the complex modulus at the first resonance frequency for each tested temperature. The complex modulus values determined from dynamic tests were then compared to the complex modulus data from cyclic tension-compression tests, assuming the same shift factors are valid for both tests. The norm of the complex modulus obtained from dynamic tests is slightly higher than the one determined 
with the conventional approach using cyclic tests. The differences observed between the two tests tend to increase with temperature but remain less than about $15 \%$ over the whole reduced frequency range. For the phase angle, results from both tests are in very good agreement. Since the strain level is approximately 500 times lower in the dynamic tests, the nonlinearity of AM with the level of strain amplitude may explain a part of the differences. The presented research shows that dynamic tests, which have the great advantage of being economic and simple to perform, can be back-analysed with a very simple model and can provide the complex modulus of AM on a wide range of frequencies and temperatures with a good accuracy.

\section{References}

1. Di Benedetto, H.; Corte, J.F. Matériaux Routiers Bitumineux 2: Constitution et Propriétés Thermomécaniques des Mélanges, Lavoisier; p. 288. (In French). (2005)

2. Halvorsen, W.G.; Brown, D.L. Impulse technique for structural response frequency testing. J. Sound Vib., 11, 8-21, doi:10.1121/1.2016847. (1977)

3. ASTM: C215-08. Standard Test Method for Fundamental Transverse, Longitudinal and Torsional Frequencies of Concrete Specimens; ASCE: West Conshoshocken, PA, USA (2008)

4. Migliori, A.; Sarrao, J. Resonant Ultrasound Spectroscopy-Applications to Physics, Materials Measurements and Nondestructive Evaluation; Wiley-Interscience publication: New York, NY, USA (1997)

5. Renault, A.; Jaouen, L.; Sgard, F. Characterization of elastic parameters of acoustical porous materials from beam bending vibrations. J. Sound Vib., 330, 1950-1963, doi:10.1016/j.jsv.2010.11.013. (2010)

6. Gudmarsson, A.; Ryden, N.; Birgisson, B. Characterizing the low strain complex modulus of asphalt concrete specimens through optimization of frequency response functions. J. Acoust. Soc. Am., 132, 2304-2312, doi:10.1121/1.4747016. (2012)

7. Gudmarsson, A.; Ryden, N.; Di Benedetto, H.; Sauzéat, C.; Tapsoba, N.; Birgisson, B. Comparing Linear Viscoelastic Properties of Asphalt Concrete Measured by Laboratory Seismic and Tension-Compression Tests. J. Nondestruct. Eval., 33, 571-582, doi:10.1007/s10921-014-0253-9. (2014)

8. Gudmarsson, A.; Ryden, N.; Di Benedetto, H.; Sauzéat, C. Complex modulus and complex Poisson's ratio from cyclic and dynamic modal testing of asphalt concrete. Constr. Build. Mater., $\quad 88, \quad 20-31$, doi:10.1016/j.conbuildmat.2015.04.007. (2015)
9. Carret, J.-C.; Pedraza, A.; Di Benedetto, H.; Sauzéat, C. Comparison of the 3-dim linear viscoelastic behavior of asphalt mixes determined with tension-compression and dynamic tests. Constr. Build. Mater., 174, 529536, doi:10.1016/j.conbuildmat.2018.04.156. (2018)

10. Carret, J.-C.; Di Benedetto, H.; Sauzéat, C. Characterization of Asphalt Mixes Behaviour from Dynamic Tests and Comparison with Conventional Cyclic Tension-Compression Tests Appl. Sci., 8(11), 2117; https://doi.org/10.3390/app8112117. (2018)

11. Carret, J.-C.; Di Benedetto, H.; Sauzéat, C. Multi-modal dynamic linear viscoelastic back analysis for asphalt mixes. J. Nondestruct. Eval., 37, 35, doi:10.1007/s10921-018-0491-3. (2018)

12. Poirier, J.E.; Pouget, S.; Leroy, C.; Delaporte, B. Projets Mure et Improvmure: Bilan à miparcours. Revue Générale des Routes et de l'Aménagement 2016, 937, 38-41. (In French) (2016)

13. Gayte, P.; Di Benedetto, H.; Sauzéat, C.; Nguyen, Q. Influence of transient effects for analysis of complex modulus tests on bituminous mixtures. Road Mater. Pavement Des., 17, 271289, doi:10.1080/14680629.2015.1067246. (2015)

14. Graziani, A.; Di Benedetto, H.; Perraton, D.; Sauzéat, C.; Hofko, B.; Poulikakos, L.; Pouget, S. Recommendation of RILEM TC 237-SIB on complex Poisson's ratio characterization of bituminous mixtures. Mater. Struct., 50, 142. (2017)

15. Perraton, D.; Di Benedetto, H.; Sauzéat, C.; Hofko, N.; Graziani, A.; Nguyen, Q. 3 Dim experimental investigation of linear viscoelastic properties of bituminous mixtures. Mater. Struct., 49, 4813-4829, doi:10.1617/s11527016-0827-3. (2016)

16. Di Benedetto, H.; Olard, F.; Sauzéat, C.; Delaporte, B. Linear viscoelastic behavior of bituminous materials: From binders to mixtures. Road Mater. Pavement Des., 5, 163-202, doi:10.1080/14680629.2004.9689992. (2004)

17. Nguyen, H.M.; Pouget, S.; Di Benedetto, H.; Sauzéat, C. Time-temperature superposition principle for bituminous mixtures. Eur. J. Environ. Civ. Eng., 13, 1095-1107, doi:10.1080/19648189.2009.9693176. (2009)

18. Nguyen, M.L.; Sauzéat, C.; Di Benedetto, H.; Tapsoba, N. Validation of the time-temperature superposition principle for crack propagation in bituminous mixtures. Mater. Struct., 46, 10751087, doi:10.1617/s11527-012-9954-7. (2013)

19. Nguyen, Q.T.; Di Benedetto, H.; Sauzéat, C.; Tapsoba, N. Time-temperature superposition principle validation for bituminous mixes in the linear and nonlinear domain. ASCE J. Mater. 
Civ. Eng., 25, 1181-1188, doi:10.1061/(ASCE)MT.1943-5533.0000658. (2013)

20. Ferry, J.D. Viscoelastic Properties of Polymers, 3rd ed.; John Wiley \& Sons: New York, NY, USA. (1980)

21. Airey, G.; Rahimzadeh, B. Combined bituminous binder and mixture linear rheological properties. Constr. Build. Mater., 18, 535-548, doi:10.1016/j.conbuildmat.2004.04.008. (2004)

22. Nguyen, Q.T.; Di Benedetto, H.; Sauzéat, C. Linear and nonlinear viscoelastic behavior of bituminous mixtures. Mater. Struct., 48, 23392351, doi:10.1617/s11527-014-0316-5. (2015)

23. Mangiafico, S.; Babadopoulos, L.; Di Benedetto, H.; Sauzéat, C. Nonlinearity of bituminous mixtures. Mech. Time-Depend. Mater., 22, 2949, doi:10.1007/s11043-017-9350-3. (2018) 